\title{
Manejo del cultivo de hortalizas y su efecto en la sustentabilidad de un valle costero del desierto de Atacama, Chile
}

\author{
Management of vegetable crops and its effect on the sustainability of a coastal \\ valley in the Atacama desert, Chile \\ Alejandro Riquelme-Garcés, Francisco González-Vallejos, \\ Pablo Contreras-Luque, Pilar Mazuela*
}

\section{RESUMEN}

\begin{abstract}
Los valles costeros de la XV Región presentan condiciones climáticas excepcionales para el cultivo de hortalizas durante todo el año, siendo el principal proveedor de hortalizas de la zona Central durante el invierno. El alto costo de la tierra en el valle de Azapa y escasa disponibilidad de agua está obligando a que los productores de hortalizas mejoren su sistema de producción para mantener esta actividad. El cultivo bajo malla ha mejorado las condiciones fitosanitarias, lo que ha impactado positivamente la producción. El objetivo de este trabajo fue describir dos procesos de producción en el cultivo de tomate (aire libre y sistema protegido con malla) y pimiento (bajo malla) y sus efectos en la producción, eficiencia en el uso del agua y la concentración de sales que emite al medioambiente. Los resultados indican que los sistemas protegidos mejoran la eficiencia en el uso del agua y disminuyen la emisión de nutrientes al medioambiente por kg producido y se sugiere que, con nuevas técnicas de cultivo, es posible mejorar considerablemente la eficiencia en el uso de los recursos sin afectar la producción.
\end{abstract}

Palabras clave: eficiencia uso del agua, salinidad, valle de Azapa, tomate, pimiento, primor.

\begin{abstract}
The coastal valleys of the XV Region of Chile have exceptional climatic conditions for vegetable cultivation during the entire year; this region is the main supplier of vegetables to the central part of the country during winter. The high cost of land in the Azapa Valley and the scarce availability of water have obliged vegetable producer to improve their production systems to maintain this activity. Cultivation under nets has improved the phytosanitary conditions of crops and impacted production positively. The objective of this study was to describe two production processes of tomato cultivation (open air and protected with net) and pepper (under netting) and their effects on production, water use efficiency and the concentration of salts which are returned to the environment. The results indicate that protected systems improve water use efficiency and decrease the emission of nutrients to the environment per $\mathrm{kg}$ produced. We suggest that with the new cultivation techniques it is possible to improve considerably the efficiency of resource use without affecting production.
\end{abstract}

Key words: water efficiency, salinity, Azapa valley, tomato, pepper, off-season.

\section{Introducción}

La región de Arica y Parinacota presenta condiciones climáticas excepcionales para el cultivo de hortalizas durante todo el año, siendo el principal proveedor de hortalizas para el país durante el invierno (Saavedra y Tapia, 2009). Predominan las condiciones de clima de desierto costero con nubosidad abundante, ausencia de heladas, vientos moderados, con alta humedad relativa y alta radiación solar directa, durante todo el año. La temperatura media anual es de $18^{\circ} \mathrm{C}$, las máximas medias anuales de $23,6^{\circ} \mathrm{C}$ y mínimas de $13,8^{\circ} \mathrm{C}$ (Torres y Acevedo, 2008). Pese al clima benigno que permite la producción de hortalizas durante todo el año, para los agricultores no es atractivo producir durante el verano principalmente por el menor precio que obtienen al aumentar la oferta por la concentración de producción durante el verano de las zonas productoras de hortalizas desde la región de Coquimbo al Maule. Esta mayor oferta desde los centros productivos más

\footnotetext{
1 Departamento de Producción Agrícola, Universidad de Tarapacá, Arica, Chile.

* Autor correspondiente: pmazuela@uta.cl
} 
próximos a la zona central, se suma al mayor costo de transporte de los productos de la comuna de Arica hacia los centros de consumo del país. Sin embargo, hay una tendencia a mejorar los procesos de producción especialmente en los sistemas de protección de cultivos y mayor tecnología de riego que permitan mantener las plantas en buenas condiciones fitosanitarias y se estima que en el valle de Azapa ya existen cerca de 500 hectáreas de cultivo bajo protección. Los sistemas de cultivo bajo malla disminuyen el uso de pesticidas, permiten la utilización de abejorros para la polinización y mantienen el cultivo en óptimas condiciones con lo que aumenta el calendario comercial de los cultivos.

Las hortalizas de mayor valor económico por superficie cultivada, uso intensivo de insumos y mano de obra son tomate para consumo fresco, que supera las 840 ha, y pimiento, con 138 ha (INE, 2008). Los rendimientos medios superan significativamente las medias nacionales, como se observa en la Tabla 1.

La demanda de los consumidores por productos de calidad ha generado cambios en el manejo de cultivos para optimizar la producción y calidad de las hortalizas. Los mercados requieren diferenciarse de sus competidores por el mayor conocimiento y acceso a la información que tienen los consumidores. Estos no solamente demandan productos de calidad sino, además, valoran que los procesos de producción sean sustentables desde el punto de vista del cuidado del medioambiente. La sostenibilidad o sustentabilidad agrícola, es un término para expresar la necesidad de generar condiciones que permitan que esta actividad pueda permanecer durante el tiempo. La agricultura depende del medio natural y causa un gran impacto en la biosfera por lo que requiere ser sometida a normativas para disminuir el impacto en esta actividad. Incentivar normativas como ChileGAP, de certificación de las Buenas Prácticas Agrícolas e ISO 14.000, de gestión ambiental; permite controlar los procesos de producción y la trazabilidad de los alimentos. Los cultivos intensivos, especialmente las hortalizas, son los más vulnerables a la sustentabilidad por el uso de recursos como agua e insumos y el efecto que estos causan en el cuidado del medioambiente.

En el caso del valle de Azapa, la instalación de la industria semillera ha generado una mayor competencia por los recursos naturales, especialmente suelo y agua. En pocos años, el costo de la tierra agrícola se ha cuadruplicado, lo que obliga a mejorar los sistemas productivos para hacer sostenible esta actividad. La sobreexplotación del acuífero del San José está afectando la disponibilidad y calidad de agua debido a: 1) habilitación de suelos; 2) ineficiencia en las técnicas de riego y 3) sobrefertilización. El acuífero del río San José, además, es la fuente de agua potable para la ciudad de Arica. Según la Dirección General de Aguas (DGA), la explotación del acuífero de Azapa está sobre su capacidad sustentable, del orden de $7001 \mathrm{seg} .^{-1}$, pues los derechos de aprovechamiento de agua superan los 3.0001 seg. ${ }^{-1}$ y la explotación real es de 1.0001 seg. ${ }^{-1}$.

Tabla 1 Superficie (ha), total nacional y regional; rendimiento $\left(\mathrm{kg} \mathrm{m}^{-2}\right)$, media nacional y media regional para cultivo de tomate y pimiento.

\begin{tabular}{lrrrr}
\hline \multirow{2}{*}{ Región } & \multicolumn{2}{c}{ Tomate } & \multicolumn{2}{c}{ Pimiento } \\
\cline { 2 - 4 } \cline { 3 - 5 } Nacional & \multicolumn{1}{c}{ ha } & ${ }^{2} \mathrm{~kg} \mathrm{~m}^{-2}$ & ${ }^{1}$ ha & ${ }^{2} \mathrm{~kg} \mathrm{~m}^{-2}$ \\
XV & 6.309 & 7,11 & 1.567 & 3,70 \\
III & 840 & 11,29 & 138 & 4,65 \\
IV & 212 & 6,16 & 22 & 4,25 \\
V & 358 & 3,08 & 601 & 3,25 \\
VI & 1.179 & 9,44 & 127 & 3,50 \\
VII & 1.062 & 5,87 & 333 & 4,22 \\
VIII & 938 & 6,89 & 116 & 4,59 \\
RM & 467 & 4,97 & 1 & 3,30 \\
\hline
\end{tabular}

Fuente: ${ }^{1}$ INE, 2008, ${ }^{2}$ INE, 2010. 


\section{Material y Método}

\section{Localización y descripción de los predios}

Para el cultivo de tomate se seleccionaron 14 agricultores de la parte alta media y baja del valle de Azapa que cultivaran, simultáneamente, tomate al aire libre y bajo malla. Con esto se aseguró que la descripción de ambos sistemas de producción descritos fuera según la tecnología utilizada y no al agricultor. En el caso del pimiento, los cultivos al aire libre fueron marginales y poco representativos por lo cual se describió el sistema de producción bajo plástico basándose en los datos de cinco productores de la parte media y baja del valle.

\section{Período descrito}

Los datos corresponden a la descripción de la campaña de 2011 y 2012 y se presentan las medias de ambas campañas. La producción es de contraestación para abastecer la demanda de estas hortalizas durante el invierno y su período de cultivo principal es de marzo a octubre.

\section{Manejo del cultivo}

El marco de plantación, riego, fertilización, tratamientos fitosanitarios fue característico para cada unidad productiva y muy heterogéneos entre sí. Para homologar criterios, se consideró la densidad de plantación, consumo hídrico, producción y emisión de iones por metro cuadrado.

\section{Análisis de fertirriego}

Se realizó un análisis de agua de riego según se utilizó agua del canal Lauca y/o de pozo. Semanalmente se analizaron muestras del fertirriego y los drenajes para determinar el consumo hídrico, la absorción de nutrientes y la emisión de iones al medioambiente.

Para obtener la información del volumen de agua aportado y drenaje, en cada unidad productiva se colocaron dos goteros control y cuatro contenedores con fibra de coco sobre una bandeja de drenaje. Cada contenedor tenía un metro de largo y un volumen de 301 , con dos a cuatro plantas por contenedor, según el marco de plantación de cada agricultor. Los datos consideraron la media de dos mediciones semanales. La muestra del gotero control y de drenajes fue analizada en el laboratorio de aguas de la Facultad de Ciencias Agronómicas de la Universidad de Tarapacá.

\section{Parámetros medidos}

Se cuantificó producción total, eficiencia en el uso del agua y emisión de iones al medioambiente. Para el cálculo de la eficiencia en el uso del agua se hizo la relación entre aporte hídrico total del período y producción total.

\section{Análisis de aniones}

Los aniones $\left(\mathrm{Cl}^{-}, \mathrm{NO}_{3}{ }^{-}, \mathrm{H}_{2} \mathrm{PO}_{4}^{-}\right.$y $\left.\mathrm{SO}_{4}{ }^{2-}\right)$ fueron determinados por cromatografía iónica con un cromatógrafo iónico DIONEX 2000 i/sp. Se ha usado la columna Ion Pac AS4A P/N 37401, la cual está protegida por una precolumna de las mismas características que la propia columna y se han empleado las siguientes condiciones cromatográficas: Weib, 1983; Engels y Marschner, 1993; Gil de Carrasco et al., 1994; Schurr y Schulze, 1995.

\section{Análisis de cationes}

Los cationes $\mathrm{Ca}^{2+} \mathrm{y} \mathrm{Mg}^{2+}$ fueron medidos por absorción atómica con un espectrofotómetro de absorción atómica marca Perkin-Elmer modelo 2380. $\mathrm{El} \mathrm{Na}^{+}$y K${ }^{+}$fueron medidos por fotometría de llama usando el mismo instrumento anterior.

\section{Emisión al medioambiente}

Para el cálculo de la emisión de iones al medioambiente se sumó la media de concentración de cationes y aniones por peso equivalente y se multiplico por el volumen medio de drenajes durante todo el año productivo.

\section{Resultados}

Los resultados de producción, consumo hídrico y emisión de iones al medioambiente se indican en la Tabla 2.

Se observa que los resultados de producción en tomates aumentan considerablemente en cultivos protegidos debido a que la planta está en mejores condiciones fitosanitarias, por lo tanto, se evita el envejecimiento prematuro de la planta observado en las plantas al aire libre, consecuentemente, aumenta 
Tabla 2. Producción media $\left(\mathrm{kg} \mathrm{m}^{-2}\right)$, eficiencia en el uso del agua (Kg producidos $\mathrm{m}^{-3}$ agua aportado) y emisión de iones al medioambiente $\left(\mathrm{T} \mathrm{ha}^{-1} \mathrm{año}^{-1}\right)$, según cultivo y sistema de cultivo.

\begin{tabular}{lrcrc}
\hline & \multicolumn{2}{c}{ Tomate } & & Pimiento \\
\cline { 2 - 3 } & Aire libre & Malla antiáfido & & Plástico \\
\hline Producción & 7,60 & 20,60 & 11,00 \\
Eficiencia en uso del agua & 12,50 & 25,60 & 13,60 \\
Emisión iones & 6,05 & 8,82 & 8,03 \\
\hline
\end{tabular}

el calendario comercial en el cultivo protegido bajo malla. Las medias de producción al aire libre son semejantes a las medias nacionales y menor a la media regional según lo indicado por el INE (2010). Sin embargo, las medias de producción en tomate bajo malla, prácticamente duplican la media regional (Tabla 1). En el caso del pimiento, la producción media del cultivo de pimiento duplica la media observada por el INE (2010).

Respecto de la eficiencia en el uso del agua, en el caso del tomate, si se calcula el inverso de la eficiencia hídrica (l aportados por kg producidos), se observa que al aire libre se requieren 801 de agua por cada $\mathrm{kg}$ producido. Estos datos son similares a los observados por Villavicencio et al., 2013, donde se indica una huella hídrica referencial de $89,11 \mathrm{~kg}^{-1}$, en la zona alta de la cuenca del río San José; $69,41 \mathrm{~kg}^{-1}$, en la zona media y $95,61 \mathrm{~kg}^{-1}$, en la zona baja, dando una media de $84,71 \mathrm{~kg}^{-1}$, en la cuenca del San José. Estos valores son menores a los descritos para otras zonas del país y se explican debido a la producción que se concentra durante el otoño-invierno con una menor demanda evapotranspirativa del cultivo, respecto de cultivos de primavera-verano. En el caso del cultivo de tomate bajo malla, la eficiencia en el uso del agua aumenta principalmente por las mejores condiciones de cultivo, menor evapotranspiración y aumento del período de cultivo. Sin embargo, este valor es menor a los $31,9 \mathrm{~kg} \mathrm{~m}^{-3}$, observados por Torres (1998) y podría explicarse por la frecuencia y tiempo de aplicación de riego, donde se ha generalizado uno a dos riegos largos diarios. Por lo tanto, se puede mejorar significativamente perfeccionando las técnicas de riego (no necesariamente los sistemas).

En el caso del cultivo de pimiento, la eficiencia en el uso del agua es mayor a lo indicado por Fernández-Zamudio y Caballero (2006) para un invernadero tipo parral básico, en suelo y sin calefacción, con eficiencia de uso de agua de 11,87 $\mathrm{kg} \mathrm{m}^{-3}$, donde el óptimo descrito es de $15,83 \mathrm{~kg} \mathrm{~m}^{-3}$, en un invernadero multitúnel; con 4,5 m a la canal; cultivo en sustrato y con calefacción permanente de $18-19^{\circ} \mathrm{C}$ de agua caliente distribuida por los raíles.

Las mediciones de campo que se han realizado a lo largo de todo el valle de Azapa por los agrónomos de la Universidad de Tarapacá demuestran que la fertilización de hortalizas es una de las prácticas culturales que más afectan la sustentabilidad económica y medioambiental del productor. $\mathrm{La}$ emisión de aniones se refiere a la carga de fertilizantes que se vierten al suelo y al acuífero, que no son aprovechados por la planta. Es mayor en sistemas productivos bajo protección debido a un período productivo más largo y, por lo tanto, aumento en la aplicación de fertilizantes. La emisión de iones total incluye la concentración de sales aportadas por el agua de riego (sodio, calcio, cloruros y sulfatos).

\section{Conclusión}

Se puede aumentar la sustentabilidad de los sistemas productivos, tanto en tomate como en pimiento, con técnicas de riego y una adecuada nutrición de las plantas. Se mejora la eficiencia en el uso del agua con técnicas de riego según las características físicas del suelo, las condiciones ambientales y los requerimientos del cultivo. $\mathrm{La}$ emisión de iones al medioambiente puede disminuir si la nutrición de las plantas se hace en base a un programa de fertilización acorde con el tipo de agua, los requerimientos del cultivo, estado fenológico de la planta, condiciones medioambientales y los objetivos de producción. La forma como se fertiliza en el valle de Azapa incide en la salinización de los suelos y afecta la calidad del agua del acuífero al lixiviar las sales.

\section{Agradecimientos}

Este trabajo ha sido financiado por el proyecto FONDEF D10R1026. 


\section{Referencias}

Dirección General de Aguas

2008. Las Mesas de Agua y las experiencias recientes del MOP en el ámbito de la gestión del agua. DGA, Región de Arica y Parinacota, 9 pp.

Engels, C.; Marschner, H.

1993. Influence of the Form of Nitrogen Supply on Root Uptake and Translocation of Cations in the Xylem Exudates of Maize (Zea mays L.), Journal of Experimental Botany, 44: 1695-1701.

Gil de Carrasco, C.; Guzmán, M.; Lorente, F.A.; Urrestarazu, M. 1994. Xylem sap extraction: a method. Commun. Soil Sci. Plant Anal., 25: 1829-1839.

Instituto Nacional de Estadísticas 2007. VII Censo Agropecuario y Forestal 2006-2007. Resultados preliminares. INE Ediciones, Santiago de Chile, 444 pp.

Instituto Nacional de Estadísticas

2010. Información Hortícola. Publicación Especial 20082009. INE Ediciones, Santiago de Chile, 128 pp.

Fernández-Zamudio, M.A.; Caballero, P.

2006. La necesidad de cuantificar la sostenibilidad de los invernaderos. Vida Rural, 13 (239): 49-54.

Saavedra, A.; Tapia, L.

2009. Evaluación de las estrategias de producción de tomate (Lycopersicon esculentum Mill) fuera de estación para la agricultura de la provincia de Arica, Chile, entre los años 1995-2005. Idesia, 27 (2): 91-96.

Schurr, U.; Schulze, E.D.

1995. The concentration of xylem sap constituents in root exudate, and on sap from intact, tranpiring castor bean plants (Ricinus communis L.), Plant, Cell and Environment, 18: 409-420.

Torres, A.

1998. Programa de investigación en parcelas experimentales cuenca del río San José, Ministerio de Obras Públicas. Direccción General de Aguas, Departamento de Estudios y Proyectos, Universidad de Tarapacá. Instituto de Agronomía, SIT N 52.

Torres, A.; Acevedo, E.

2008. El problema de salinidad en los recursos suelo y agua que afectan el riego en los valles de Lluta y Azapa en el norte de Chile. Idesia, 26 (3): 31-44.

Villavicencio, A; Riquelme, P; Pérez, A.

2013. Huella hídrica en tomate fresco, pp. 87-92. En: Determinación de la huella del agua y estrategias de manejo de recursos hídricos. Osorio, A. (Ed). Series Actas INIA $\mathrm{N}^{\circ}$ 50. La Serena, Chile.

Weib, J.

1983. Einführung in die Inoenchromatograpie. CLB-Chemie für Labor und Betrieb, 41: 103-104. 
